\title{
Effects of low-intensity pulsed ultrasound (LIPUS)-pretreated human amnion-derived mesenchymal stem cell (hAD-MSC) transplantation on primary ovarian insufficiency in rats
}

\author{
Li Ling ${ }^{1}$, Xiushan Feng ${ }^{1}$, Tianqin Wei ${ }^{1}$, Yan Wang ${ }^{2}$, Yaping Wang ${ }^{3}$, Wenqian Zhang ${ }^{1}$, Lianli He ${ }^{4}$, Ziling Wang ${ }^{1}$, \\ Qianru Zeng ${ }^{1}$ and Zhengai Xiong ${ }^{1 *}$
}

\begin{abstract}
Background: Human amnion-derived mesenchymal stem cells (hAD-MSCs) have the features of mesenchymal stem cells (MSCs). Low-intensity pulsed ultrasound (LIPUS) can promote the expression of various growth factors and anti-inflammatory molecules that are necessary to keep the follicle growing and to reduce granulosa cell (GC) apoptosis in the ovary. This study aims to explore the effects of LIPUS-pretreated hAD-MSC transplantation on chemotherapy-induced primary ovarian insufficiency (POI) in rats.

Methods: The animals were divided into control, POI, hAD-MSC treatment, and LIPUS-pretreated hAD-MSC treatment groups. POI rat models were established by intraperitoneal injection of cyclophosphamide (CTX). The hAD-MSCs isolated from the amnion were exposed to LIPUS or sham irradiation for 5 consecutive days and injected into the tail vein of POI rats. Expression and secretion of growth factors promoted by LIPUS in hAD-MSCs were detected by real-time quantitative polymerase chain reaction (RT-gPCR) and enzyme-linked immunosorbent assay (ELISA) in vitro. Estrous cycle, serum sex hormone levels, follicle counts, ovarian pathological changes, GC apoptosis, Bcl2 and Bax expression, and pro-inflammatory cytokine levels in ovaries were examined.

Results: Primary hAD-MSCs were successfully isolated from the amnion. LIPUS promoted the expression and secretion of growth factors in hAD-MSCs in vitro. Both hAD-MSC and LIPUS-pretreated hAD-MSC transplantation increased the body and reproductive organ weights, improved ovarian function, and reduced reproductive organ injuries in POI rats. Transplantation of hAD-MSCs increased the BCl-2/Bax ratio and reduced GC apoptosis and ovarian inflammation induced by chemotherapy in ovaries. These effects could be improved by pretreatment with LIPUS on hAD-MSCs.

Conclusion: Both hAD-MSC transplantation and LIPUS-pretreated hAD-MSC transplantation can repair ovarian injury and improve ovarian function in rats with chemotherapy-induced POI. LIPUS-pretreated hAD-MSC transplantation is more advantageous for reducing inflammation, improving the local microenvironment, and inhibiting GC apoptosis induced by chemotherapy in ovarian tissue of POI rats.
\end{abstract}

Keywords: Low-intensity pulsed ultrasound (LIPUS), Human amnion-derived mesenchymal stem cells (hAD-MSCS), Primary ovarian insufficiency/failure (POI/POF), Chemotherapy, Growth factors

\footnotetext{
* Correspondence: xza20170407@163.com

'Department of Obstetrics and Gynecology, the Second Affiliated Hospital of

Chongqing Medical University, No. 76, Linjiang Road, Chongqing 400010,

China

Full list of author information is available at the end of the article
} 


\section{Background}

Primary ovarian insufficiency (POI), also known as premature ovarian failure (POF), is pathologically characterized by oligomenorrhea or amenorrhea, low levels of gonadal hormones, and high levels of follicle-stimulating hormone (FSH; > $25 \mathrm{mIU} / \mathrm{ml}$ ) in females before the age of 40 years [1-3]. POI induces multiple health risks, and affects about $1 \%$ of women under the age of 40 years [2]. Chemotherapy is a commonly used treatment method for women bearing tumors, which could induce ovarian failure, including follicle loss, vascular damage, and tissue fibrosis, especially in young females [4-7]. Therefore, it is of great importance to develop and/or improve the treatment strategies for the irreversible pathogenesis of POI.

Regenerative medicine research suggests that mesenchymal stem cell (MSC) transplantation may restore the ovarian structure and function in animal models of POI, providing an effective treatment method $[8,9]$. Recently, human amnion-derived mesenchymal stem cells (hADMSCs) have been shown to have the features of MSCs [10-12]. Self-renewal capacity, multipotency, low immunogenicity, and noninvasive application without controversy make hAD-MSCs a promising and useful source of stem cells for transplantation and regenerative medicine [11-13]. Some studies have demonstrated the efficacy of hAD-MSC transplantation for disease treatment in animal models [14-16]. However, whether hAD-MSC transplantation can restore the ovarian function in chemotherapy-induced POI is still unknown.

Low-intensity pulsed ultrasound (LIPUS) was defined as a safe and effective therapy for promoting fracture healing by the Food and Drug Administration (FDA) in 1994. However, there is currently no standard for LIPUS treatment, and studies have been conducted with intensity levels between 5 and $500 \mathrm{~mW} / \mathrm{cm}^{2}$, frequencies between $45 \mathrm{kHz}$ and $3 \mathrm{MHz}$, pulse repetition rate from $100 \mathrm{~Hz}$ to $1 \mathrm{kHz}$, and duty cycles from 20\% to 50\% [1719]. It has been reported that mechanical stimulation by LIPUS is able to induce a series of biochemical events at the cellular level that can promote the secretion of various growth factors and anti-inflammatory molecules [17, 20-22], including fibroblast growth factor (FGF)2, insulin-like growth factor (IGF)-1, and vascular endothelial growth factor (VEGF). MSCs have been shown to have the ability to sense and respond to physical stimuli $[23,24]$. However, whether LIPUS can promote the expression and secretion of those growth factors in hADMSCs are still unknown.

Apoptosis of oocyte and granulosa cells (GCs) has been identified as one possible underlying mechanism for chemotherapy-induced POI, which leads to follicular atresia and loss of ovarian reserve [25]. It has been demonstrated that, although the follicle growth is mainly regulated by pituitary gonadotropins in monovular species, mediation of some local growth factors, such as FGF2, IGF-1, hepatocyte growth factor (HGF), and VEGF, are essential to keep the follicle growing [26, 27]. Importantly, those growth factors could regulate GCs and reduce GC apoptosis [26, 28, 29]. In this study, whether LIPUS could promote the expression and secretion of those growth factors in hAD-MSCs, and whether LIPUS-pretreated hAD-MSC transplantation could be more efficient to treat patients with POI, was investigated. The efficacy of hAD-MSCs and LIPUS-pretreated hAD-MSC transplantation on chemotherapy-induced POI was evaluated in rat models.

\section{Methods}

Isolation and culture of hAD-MSCs

The research was in compliance with the Helsinki Declaration and approved by the Ethics Committee of the Second Affiliated Hospital of Chongqing Medical University. Primary hAD-MSCs were isolated from term amnion, according to the protocol described by Fang et al. [30]. Term placentas were collected from healthy donors who received cesarean section at the Second Affiliated Hospital of Chongqing Medical University, Chongqing, China. Written informed consent was obtained from all these donors before collection.

Amnion was separated from placenta and digested with $0.05 \%$ trypsin and $0.02 \%$ ethylenediaminetetraacetic acid (EDTA; Gibco, Grand Island, NY, USA) at $37{ }^{\circ} \mathrm{C}$ for $40 \mathrm{~min}$. The mixture was filtered through a 300 -mesh sieve and residues were collected (the process was repeated once). The residues were further digested with $0.75 \mathrm{mg} / \mathrm{mL}$ collagenase II (Sigma-Aldrich, St. Louis, MO, USA) and $0.075 \mathrm{mg} / \mathrm{mL}$ DNase I (Worthington, New Jersey, USA) at $37^{\circ} \mathrm{C}$ for $1.5 \mathrm{~h}$. After filtration and centrifugation, the isolated hAD-MSCs were cultured in the L-DMEM medium (Gibco, Grand Island, NY, USA), supplemented with $10 \%$ fetal bovine serum (FBS; Invitrogen, Carlsbad, CA, USA), $100 \mathrm{U} / \mathrm{mL}$ penicillin, and $0.1 \mathrm{mg} / \mathrm{mL}$ streptomycin (Beyotime, Haimen, Jiangsu, China), in a humidified atmosphere with $5 \% \mathrm{CO}_{2}$ at $37{ }^{\circ} \mathrm{C}$. Cells were subcultured at a ratio of $1: 2$ when 90-100\% confluency was reached. The third to fifth passages (P3-5) of hAD-MSCs were used for the following experiments.

\section{Identification and characterization of hAD-MSCs}

Primary hAD-MSCs were identified and characterized by flow cytometry. In total, $1 \times 10^{6}$ cells were incubated with fluorescein isothiocyanate (FITC)-labeled antihuman antibodies FITC-CD105, FITC-CD45, FITCCD34, FITC-CD19, FITC-CD14, FITC-HLA-DR, and FITC-CD90, phycoerythrin (PE)-labeled PE-CD73, or FITC- or PE-conjugated isotype control (all from $\mathrm{BD}$ 
Biosciences, San Diego, CA, USA), and analyzed on a FACSCalibur flow cytometer (BD Biosciences). Data were processed using the Cell Quest software (BD Biosciences).

To identify the multipotent differentiation of hADMSCs, the cells were cultured in osteogenic differentiation medium, adipogenic differentiation medium, and chondrogenic differentiation medium, respectively, for 21 days. After staining with Alizarin Red S, Oil Red O, and Alician blue (all from Cyagen Biosciences Inc., Suzhou, Jiangsu, China), respectively, the cells were observed under an inverted microscope (Olympus Corporation, Tokyo, Japan).

\section{LIPUS protocol}

The LIPUS exposure device (Chongqing Haifu Medical Technology Co. Ltd., Chongqing, China) consisted of six transducers (34.8 $\mathrm{mm}$ in diameter) in an array, which was specifically designed for the six-well culture plate. LIPUS parameters were set as follows: frequency, $0.25 \mathrm{MHz}$; burst width sine wave, $200 \mu \mathrm{s}$; duty cycle, $20 \%$; pulse repetition frequency, $1 \mathrm{kHz}$; spatial-average temporal-average intensity $\left(\mathrm{I}_{\mathrm{SATA}}\right), 30 \mathrm{~mW} / \mathrm{cm}^{2}$; and exposure time, $30 \mathrm{~min}$. The conditions have previously been proven to benefit the viability of hAD-MSCs according to our preliminary experiments (data not shown).

\section{Labeling and tracking of hAD-MSCs}

To track and locate the transplanted hAD-MSCs in the ovarian tissues, the cells were pre-labeled with the PKH26 Red Fluorescent Cell Linker Kits (Sigma-Aldrich, St. Louis, MO, USA) [31, 32]. The PKH26-labeled hADMSCs were transplanted into rats via the tail vein. At $24 \mathrm{~h}, 4$ weeks, and 8 weeks after cell transplantation, ovaries were fixed with optimal cutting temperature (OCT) compound and made into fresh sections (10 $\mu \mathrm{m}$ thick). After fixation with pre-cooled acetone for $10 \mathrm{~min}$, the sections were washed and incubated with 2-(4-amidinophenyl)-6-indolecarbamidine dihydrochloride (DAPI; Boster Biological Technology Co. Ltd., Wuhan, Hubei, China) at room temperature for $10 \mathrm{~min}$. The sections were then imaged under a fluorescent microscope (Nikon Corporation, Tokyo, Japan).

\section{CCK-8 assay}

The cell counting kit- 8 (CCK-8) assay (Beyotime) was used to detect the growth of hAD-MSCs according to the manufacturer's instructions. Cells were seeded onto the 96-well plate at a density of $5 \times 10^{3}$ cells/well and cultured in the incubator. The optical density (OD) value at $450 \mathrm{~nm}$ was then measured every day for 4 continuous days using a plate reader (1510 model; Thermo Fisher Scientific Oy, Vantaa, Finland).

\section{Animal model establishment and grouping}

In total, 160 female Sprague-Dawley (SD) rats (10-12 weeks of age) were purchased from the Experimental Animal Center of Chongqing Medical University (Chongqing, China). Animal experimental protocols were approved by the Ethics Committee of the Second Affiliated Hospital of Chongqing Medical University. These animals were randomly divided into control, POI, hAD-MSC treatment (hAD-MSCs), and LIPUS-pretreated hAD-MSC treatment (LIPUS + hAD-MSCs) groups ( $n=40$ each group). To establish the POI model, the rats from the POI, hAD-MSCs, and LIPUS + hAD-MSCs groups received intraperitoneal injection of cyclophosphamide (CTX; Hengrui medicine Co. Ltd., Lianyugang, Jiangsu, China; re-suspended in normal saline), at a dose of $50 \mathrm{mg} / \mathrm{kg}$ on the first day and then a daily dose of $8 \mathrm{mg} / \mathrm{kg}$ for the next 14 consecutive days [33]. The rats from the control group were only injected with normal saline. At $24 \mathrm{~h}$ after chemotherapy, the rats from the hAD-MSCs and LIPUS + hAD-MSCs groups were injected with $0.6 \mathrm{~mL}$ phosphate-buffered saline (PBS) containing $4 \times 10^{6}$ hAD-MSCs and LUPUSpretreated hAD-MSCs labeled with $\mathrm{PKH}-26$, respectively $[31,34,35]$, via the tail vein [36].

At 1 week after CTX injection, the vaginal smears of ten rats from each group were obtained at 9.00 am daily for 10 weeks to observe the estrous cycle. The rats in the stages of estrus, proestrus, metestrus, and diestrus were identified and quantified by hematoxylin and eosin (H\&E) staining.

From the first day of chemotherapy, animals in the four groups were weighed every week for 12 consecutive weeks. From 1 to 10 weeks after transplantation rats were narcotized and the organs and blood were collected. Organs were fixed with $4 \%$ paraformaldehyde or OCT compound (Sakura Finetek USA Inc., Torrance, CA, USA) for further processing. Serum was collected by centrifugation and stored at $-80^{\circ} \mathrm{C}$ for hormone tests.

\section{Real-time quantitative polymerase chain reaction (RT-qPCR)}

The hAD-MSCs were seeded onto the six-well plate at a density of $1 \times 10^{5}$ cells $/ \mathrm{mL}$. After $12 \mathrm{~h}$ the cells were exposed to sham irradiation (ultrasound generator was not switched on) or LIPUS for 5 consecutive days. The mRNA expression levels of growth factors (FGF2, IGF-1, HGF, and VEGF) were then detected by RT-qPCR.

Total RNA was extracted using TRIzol Reagent (Invitrogen, Carlsbad, CA, USA). The RNA sample was quantified and reverse transcribed into cDNA using the ReverTra Ace- $\alpha$-first strand cDNA Synthesis Kit (TOYOBO Life Science, Shanghai, China). RT-qPCR was performed using a CFX96 Real-Time PCR Detection System (Bio-Rad, Hercules, CA, USA) with SYBR Green Real-Time PCR Master Mix (TOYOBO Life Science). 
Primers used in the experiment are listed in Table 1. Target gene expression was determined using the $2^{-\Delta \Delta \mathrm{Ct}}$ method. GAPDH was used as the internal reference.

\section{TUNEL assay}

Ovarian cell apoptosis was detected by the TUNEL apoptosis assay kit (Roche Applied Science, Basel, Switzerland) according to the manufacturer's instructions. Nuclei of ovarian apoptotic cells were stained dark brown. Sections were observed and imaged by an optical microscope (Olympus Corporation, Tokyo, Japan).

\section{H\&E staining}

To analyze the ovarian morphology and follicle counts, ten ovaries from each group were collected at 1 month after hAD-MSC transplantation and fixed with $4 \%$ paraformaldehyde for $48 \mathrm{~h}$. The ovaries were dehydrated, embedded in paraffin, and cut into 5 - $\mu \mathrm{m}$ sections. After H\&E staining, sections were observed and imaged by an optical microscope (Olympus Corporation). Follicles were counted and categorized in every fifth section through the ovary [8], which were classified as primordial, primary, secondary, preovulatory, and atretic follicles, respectively $[37,38]$.

\section{Enzyme-linked immunosorbent assay (ELISA)}

Serum levels of anti-Müllerian hormone (AMH), E2, and FSH at the indicated time points $(0,2,4,6,8$, and 10 weeks after cell transplantation) were detected by ELISA kits (Uscn Life Science, Wuhan, Hubei, China) according to the manufacturer's instructions.

To detect the inflammatory cytokines in the ovaries at $24 \mathrm{~h}$ after cell transplantation, ovarian tissue was collected from the four groups and homogenized. Ovarian supernatant was collected, and the levels of interleukin (IL)-1 $\beta$, IL-6, tumor necrosis factor (TNF)- $\alpha$, and VEGF were detected by ELISA kits (all from Boster Biological

Table 1 Primer sequences for real-time quantitative polymerase chain reaction

\begin{tabular}{|c|c|c|c|}
\hline & Primers sequence $5^{\prime}-3^{\prime}$ & Amplification & Accession no. \\
\hline \multirow[t]{2}{*}{ FGF2 } & TACCCATACAGCAGCAGCCTAGC & $145 \mathrm{bp}$ & NM_002006 \\
\hline & GCCGCCTAAAGCCATATTCATTCAC & & \\
\hline \multirow[t]{2}{*}{ HGF } & GGCCAAGTCCCCAAACAATTC & $107 \mathrm{bp}$ & NM_000601 \\
\hline & GCCGCCCTATATTCTGTGGACTAAG & & \\
\hline \multirow[t]{2}{*}{ IGF1 } & CCACCACCTITCAACTIITTATCAC & $197 \mathrm{bp}$ & NM_000618 \\
\hline & CGCGTITATTCTCCATGCTCTTG & & \\
\hline \multirow[t]{2}{*}{ VEGF } & GCGCAAGAAATCCCGGTATAAG & $233 \mathrm{bp}$ & NM_001025366 \\
\hline & GCCTCGGCTTGTCACATCTG & & \\
\hline \multirow[t]{2}{*}{ GAPDH } & CTCCTCCGGGTGATGCTTTTCCTAG & $248 \mathrm{bp}$ & NM_00125679s \\
\hline & CTCGCTCCTGGAAGATGGTGATG & & \\
\hline
\end{tabular}

Technology Co., Ltd.) according to the manufacturer's instructions.

To detect the growth factors secreted by hAD-MSCs, cells were treated as described in the RT-qPCR subsection, except for that the complete medium was replaced with serum-free medium on the third day before sham irradiation or LIPUS treatment. After $72 \mathrm{~h}$, the cell supernatant was collected to detect the levels of IGF-1, HGF, and VEGF (all from Uscn Life Science) according to the manufacturer's instructions.

\section{Western blot analysis}

At 1 week after cell transplantation, ovaries were collected from the four groups and homogenized with RIPA lysis buffer (Beyotime). Protein concentration was determined by the Bradford Protein Assay Kit (Beyotime). Protein samples were separated by SDSPAGE and subsequently electrically transferred onto the PVDF membrane (Millipore, Billerica, MA, USA). After washing and blocking with 5\% skimmed milk for $1 \mathrm{~h}$, the membrane was incubated with rabbit anti-rat primary antibodies for Bcl-2 and Bax (Abcam, Cambridge, UK) at $4{ }^{\circ} \mathrm{C}$ overnight. After washing three times, the membrane was incubated with the secondary antibodies (Cell Signaling Technology, Boston, MA, USA) at room temperature for $1 \mathrm{~h}$. The BeyoECL Plus kit was used for color development according to the manufacturer's instructions.

\section{Statistical analysis}

Data are expressed as mean \pm SD. Statistical analysis was performed with the SPSS 22.0 software (IBM, Armonk, NY, USA). Independent-samples $t$ test and one-way analysis of variance (ANOVA) were used for two- and multiple-group comparisons, respectively. Statistical significance was set at $P<0.05$.

\section{Results}

\section{Identification and characterization of hAD-MSCs}

In our experiments, an average of approximately $3.08 \pm 1.36 \times 10^{7}$ hAD-MSCs could be yielded from each amnion. These hAD-MSCs displayed fibroblastic morphology at 10-12 days after isolation (Fig. 1a), and the subcultured cells also maintained fibroblastic morphology (Fig. 1b and c). The phenotype of hADMSCs (P3) was detected by flow cytometry. As shown in Fig. 1d, the phenotype characteristics of isolated hAD-MSCs were similar to bone marrowderived MSCs (BM-MSCs). Moreover, the multipotency of hAD-MSCs was confirmed by their ability to differentiate into osteoblasts, adipocytes, and chondroblasts, respectively (Fig. 1e-g). These results indicate that 

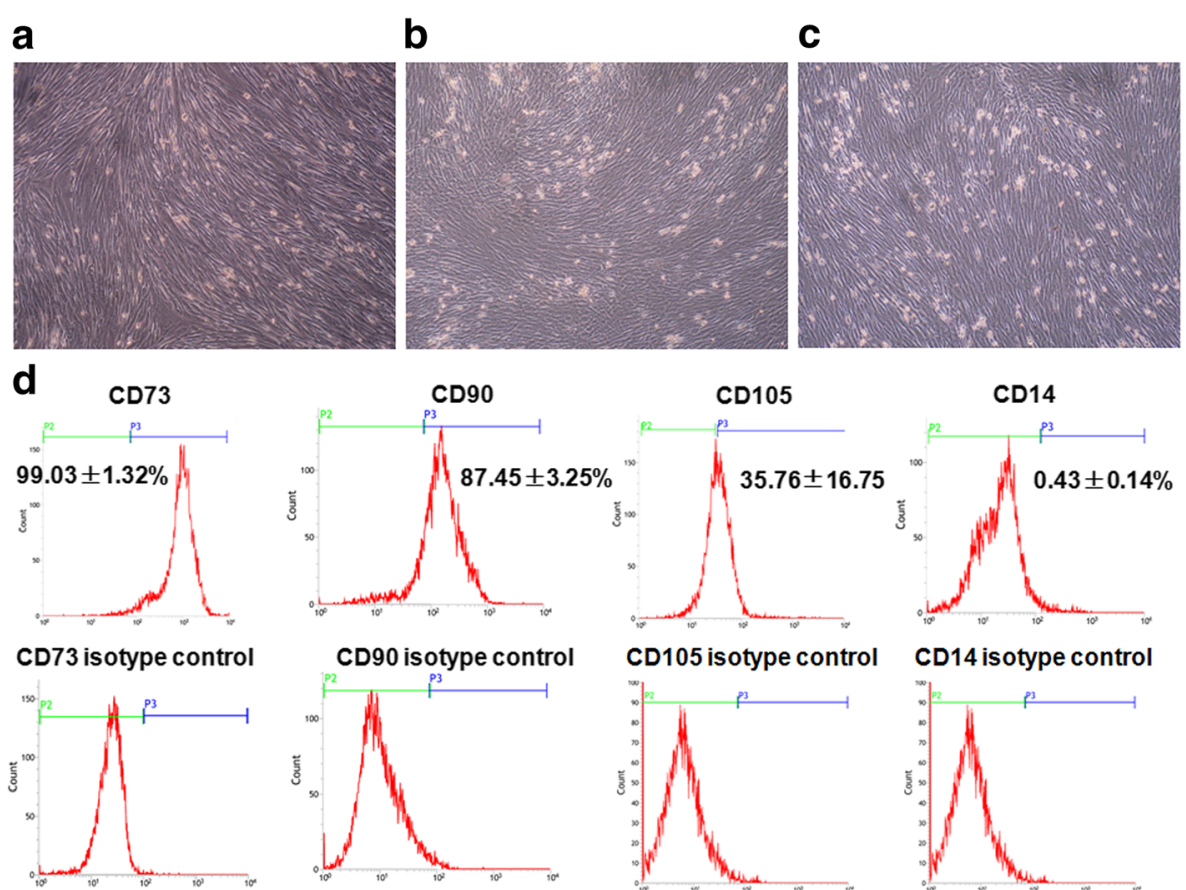

CD90 isotype control

CD105 isotype control
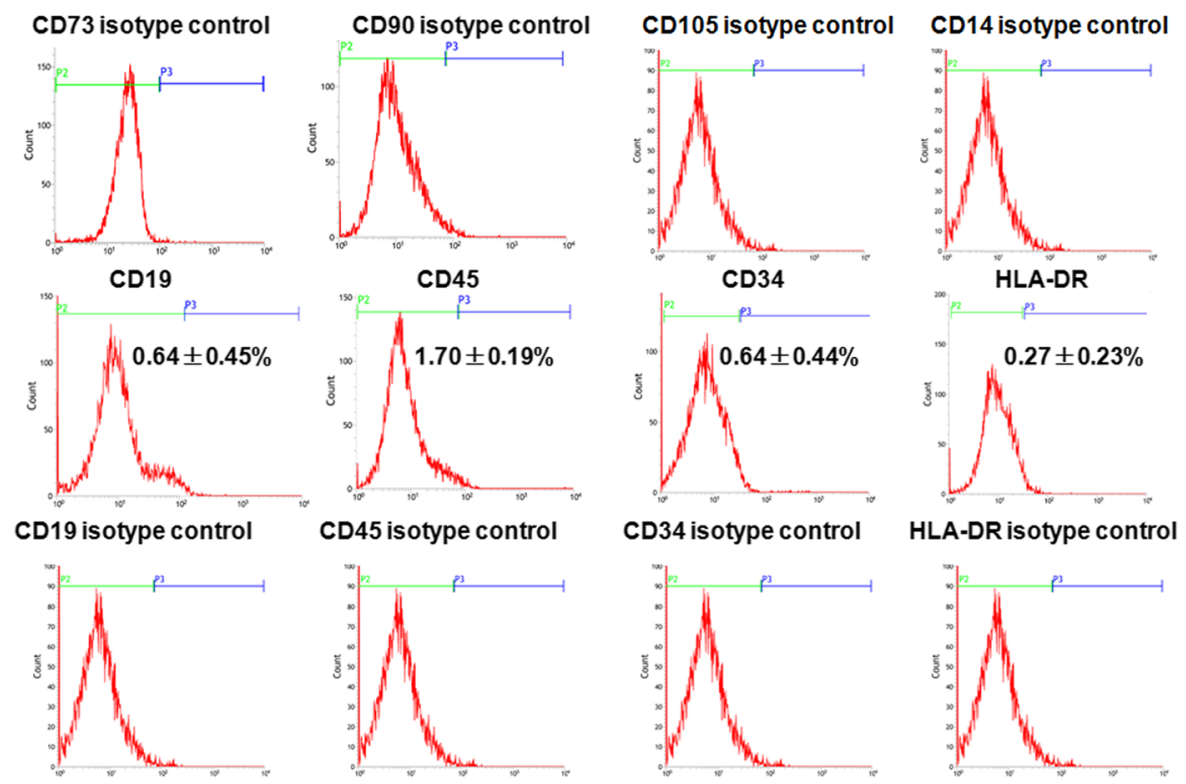

CD34 isotype control
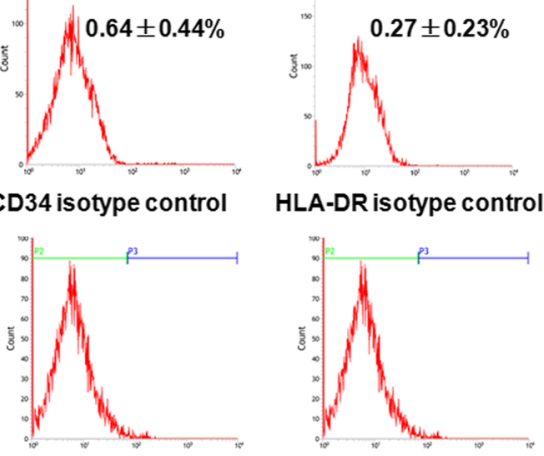

HLA-DR isotype control

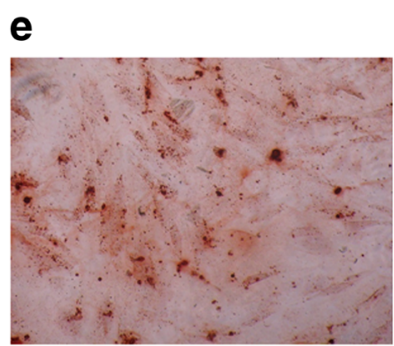

f

g
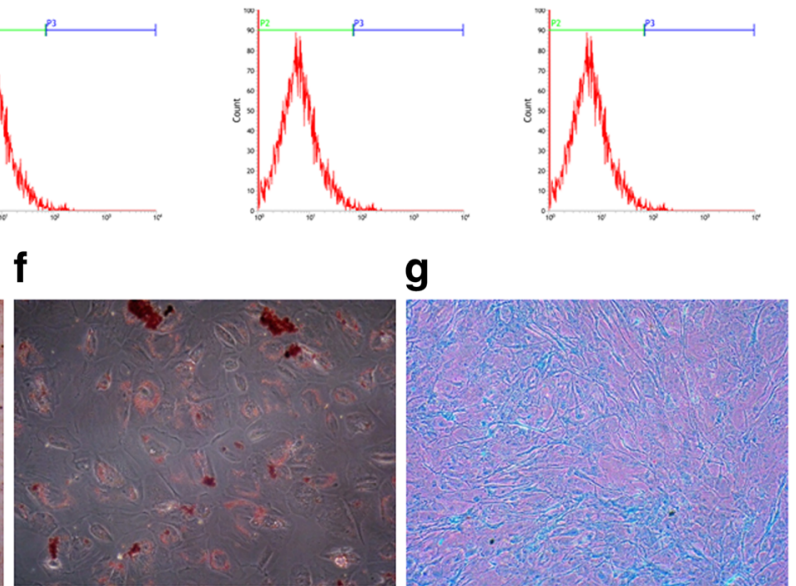

Fig. 1 Morphology, phenotype, and multipotency of hAD-MSCs. a-c The cell morphology of passages 0 (a), 3 (b), and 5 (c) hAD-MSCs (100x). d Flow cytometry analysis of cell phenotype. $\mathbf{e - g}$ The osteogenic (e; 100x), adipogenic (f; 200x), or chondrogenic (g; 100x) differentiation of hAD-MSCs was assessed by specific staining

the isolated cells are identified as hAD-MSCs, and have the common characteristics of multipotent MSCs.

\section{LIPUS promotes expression and secretion of growth factors in hAD-MSCs}

To determine whether LIPUS could promote the expression and secretion of FGF2, IGF-1, HGF, and VEGF in hAD-
MSCs, RT-qPCR and ELISA were performed. We found that the relative mRNA expression and protein secretion levels of IGF-1, HGF, and VEGF in hAD-MSCs in the LIPUS group were significantly higher than in the sham irradiation group $(P<0.05$ and $P<0.01$, respectively; Fig. $2 \mathrm{~b})$. These results demonstrate that LIPUS can promote the expression and secretion of IGF-1, HGF, and VEGF in hAD-MSCs. 

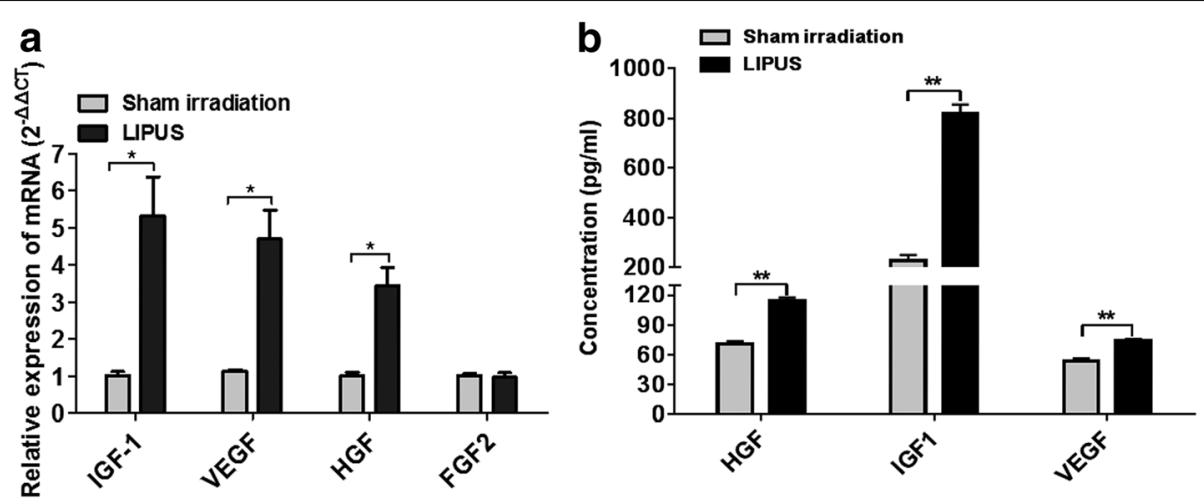

Fig. 2 LIPUS promotes growth factor expression in hAD-MSCs. a The relative mRNA expression levels of FGF2, IGF-1, HGF, and VEGF in hAD-MSCS in the LIPUS and sham irradiation groups were detected by RT-qPCR. $\mathbf{b}$ The levels of IGF-1, HGF, and VEGF secreted by hAD-MSCs in the cell supernatant in the LIPUS and sham irradiation groups were detected by ELISA. ${ }^{*} P<0.05,{ }^{*} P<0.01$. HGF hepatocyte growth factor, IGF1 insulinlike growth factor-1, LIPUS low-intensity pulsed ultrasound, VEGF vascular endothelial growth factor

\section{In vivo tracking of hAD-MSCs}

In order to track and locate the hAD-MSCs in vivo, the cells were pre-labeled with PKH26 before transplantation (Fig. 3a). As detected by flow cytometry, the cell labeling rate was $99.07 \pm 0.36 \%$ (Fig. 3b), which did not decrease after cell passaging (98.60 $\pm 0.20 \%$; Fig. 3c). Cell growth was investigated by the CCK- 8 assay. The results showed that there was no significant change in cell activity and proliferation between PKH26-labeled and unlabeled hAD-MSCs (Fig. 3d). These results demonstrate that PKH26 labeling is efficient and stable and does not influence the activity of hAD-MSCs. The location and fate of transplanted PKH26-labeled hAD-MSCs in ovarian tissue were traced at $24 \mathrm{~h}, 4$ weeks, and 8 weeks after cell transplantation (Fig. $3 \mathrm{e}-\mathrm{g}$ ). The results show that PKH26-labeled cells were only located in the interstitium of ovaries, rather than in follicles, after transplantation in both the hAD-MSCs and LIPUS + hAD-MSCs groups. Moreover, the red fluorescent signal could still be clearly observed in ovaries at 8 weeks after cell transplantation in those two groups.

\section{Transplantation of hAD-MSCs increases body and reproductive organ weights of $\mathrm{POI}$ rats}

The body and reproductive organ weights of the rats were investigated next. Our results show that, compared to the control group, the body weights of rats in the POI, hAD-MSCs, and LIPUS + hAD-MSCs groups were significantly decreased after chemotherapy $(P<0.05$; Fig. 4b). Moreover, compared to the POI group, the body weights in both the hAD-MSCs and LIPUS + hADMSCs groups were significantly increased, starting from the fourth week after cell transplantation $(P<0.05$; Fig. 4a and $b$ ). However, there was no significant difference between the hAD-MSCs and LIPUS + hAD-MSCs groups. On the other hand, ovaries and uteruses were weighed at $0,2,4,6,8$, and 10 weeks after cell transplantation.
Our results show that, compared to the POI group, the weights of ovaries and uteruses in the hAD-MSCs and LIPUS + hAD-MSCs groups were significantly increased, starting from the second week after cell transplantation $(P<0.05$; Fig. $4 \mathrm{c}-\mathrm{f})$. However, there was no significant difference between the hAD-MSCs and LIPUS + hADMSCs groups. These results demonstrate that both hAD-MSC and LIPUS-pretreated hAD-MSC transplantation can increase the body and reproductive organ weights of POI rats.

\section{Transplantation of hAD-MSCs improves ovarian function in $\mathrm{POI}$ rats}

To investigate the effects of hAD-MSCs and LUPUSpretreated hAD-MSC transplantation on ovarian function in POI rats, the estrous cycle and serum levels of $\mathrm{AMH}, \mathrm{E} 2$, and FSH were detected. The results show that $50 \%$ of rats had irregular estrous cycle in the first week after chemotherapy, and $100 \%$ of rats remained at diestrus from the third week after chemotherapy. At 1, 4, and 8 weeks after hAD-MSC transplantation, vaginal smears from the four groups were observed (Figs. 5a and b). The results show that the percentages of rats with abnormal cyclicity are decreased in both the hAD-MSCs and LIPUS + hAD-MSCs groups from the first week after cell transplantation.

On the other hand, compared to the POI group, the levels of $\mathrm{AMH}$ (indicating ovarian reserve) was significantly increased in the hAD-MSCs and LIPUS + hADMSCs groups, starting from the second week after cell transplantation $(P<0.01$; Fig. $5 \mathrm{c})$. Moreover, compared to the POI group, the levels of E2 were significantly increased in the hAD-MSCs and LIPUS + hAD-MSCs groups, starting from the eighth week after cell transplantation (Fig. 5d), while the FSH level was significantly decreased, starting from the second week after cell transplantation (Fig. 5e). However, there was no significant 


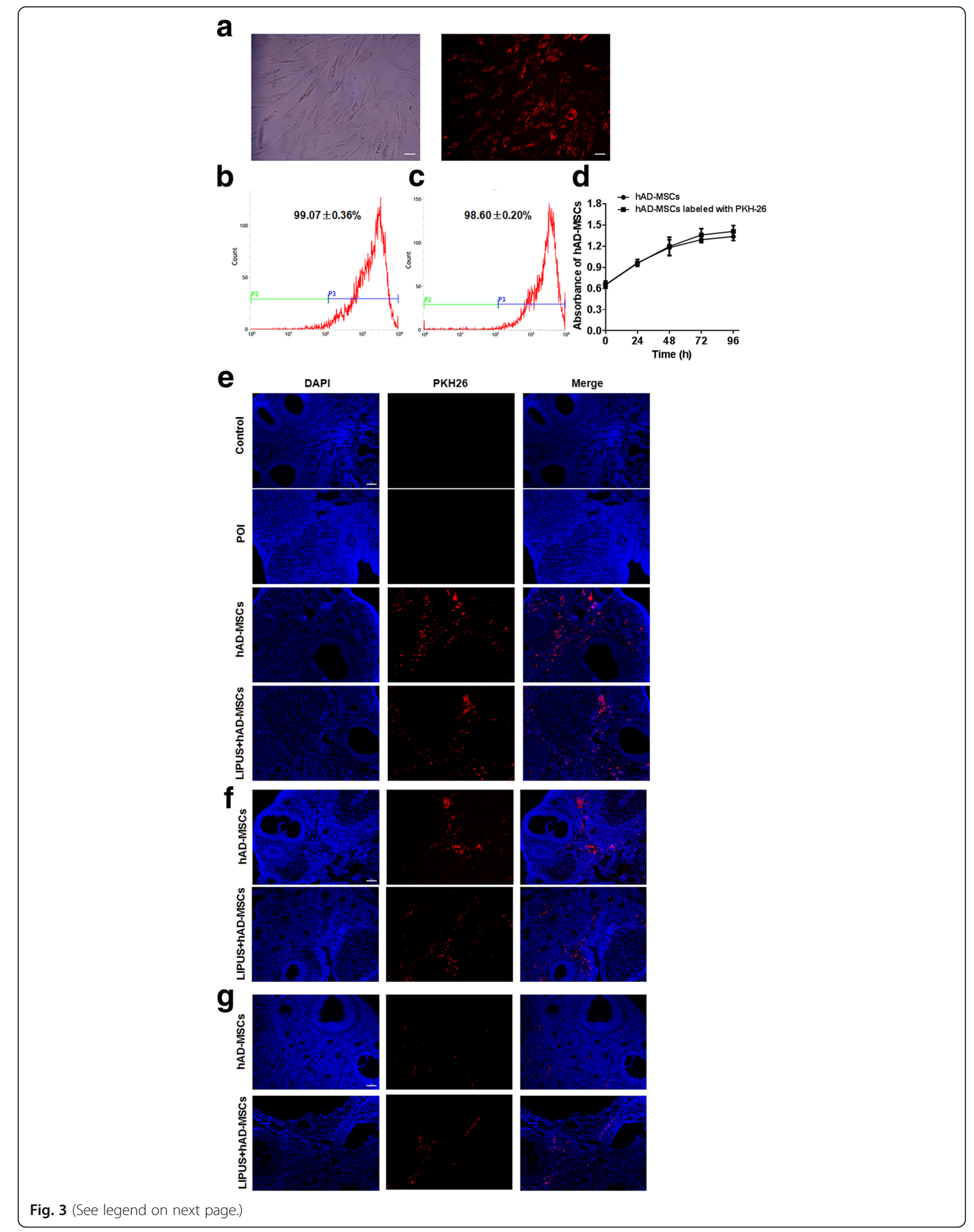


(See figure on previous page.)

Fig. 3 In vivo hAD-MSC tracking. a PKH26-labeled hAD-MSCs showed red fluorescence (100x). b,c The labeling rates of PKH26-labeled hAD-MSCS (b) and their subcultured cells (c) were detected by flow cytometry. $\mathbf{d}$ The growth curves of PKH26-labeled and unlabeled hAD-MSCS were measured by CCK-8 assay $(n=6)$. e-g Transplanted hAD-MSCs were observed at $24 \mathrm{~h}(\mathbf{e}), 4$ weeks $(\mathbf{f})$, and 8 weeks $(\mathbf{g})$ after cell transplantation in ovaries $(100 \mathrm{x})$. Scale bars $=100 \mu \mathrm{m} .{ }^{*} P<0.05$, ${ }^{*} P<0.01$. hAD-MSCs human adipose-derived mesenchymal stem cells, LIPUS low-intensity pulsed ultrasound, $P O I$ primary ovarian insufficiency

difference between the hAD-MSCs and LIPUS + hADMSCs groups. These results demonstrate that both hADMSCs and LIPUS-pretreated hAD-MSC transplantation can protect the ovarian reserve and improve the ovarian function in POI rats.
Transplantation of hAD-MSCs reduces reproductive organ injuries in POI rats

Ovaries and uteruses in the four groups were collected and subjected to pathological analysis. Our results show that there were significantly fewer primordial, primary,

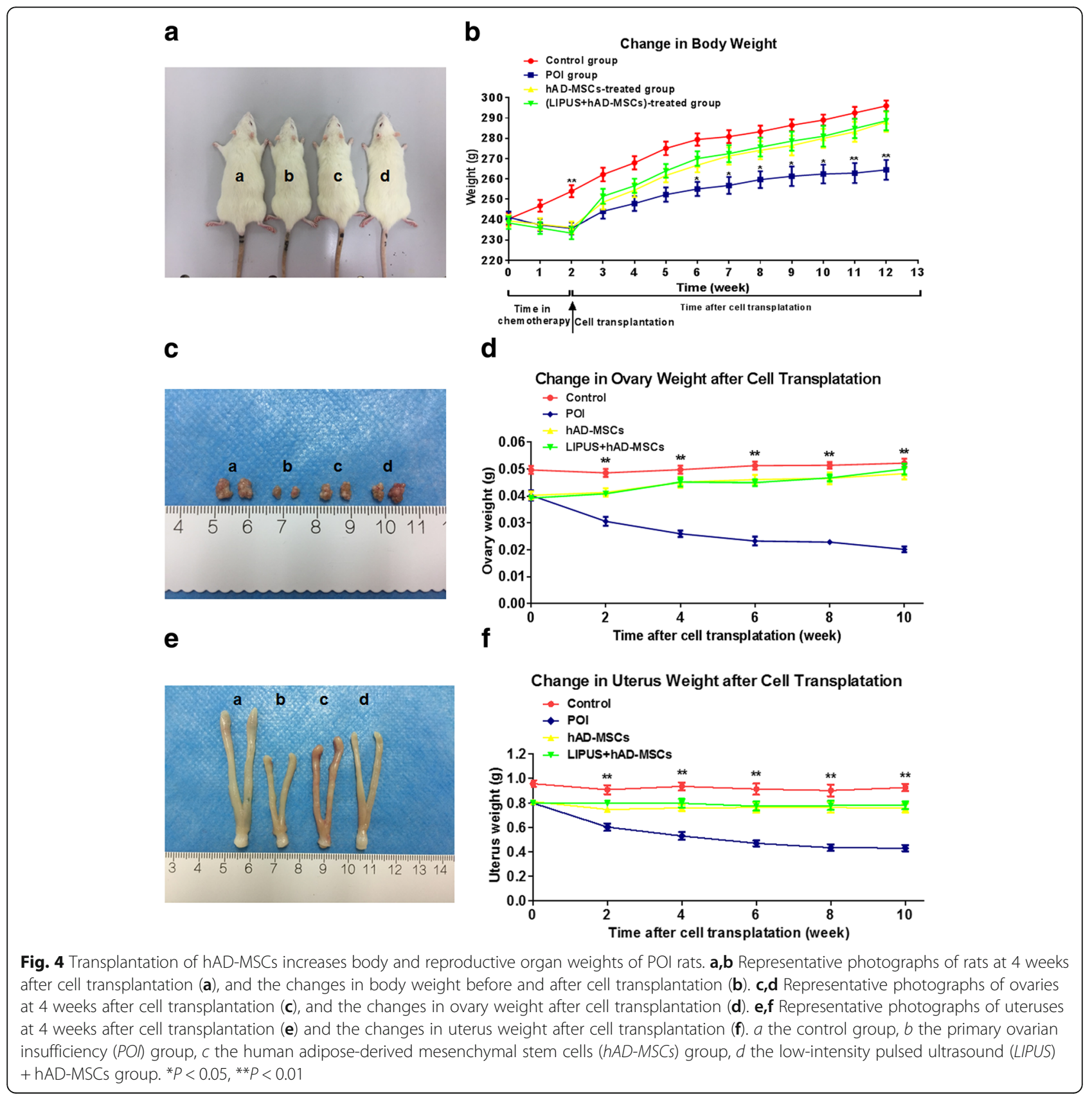



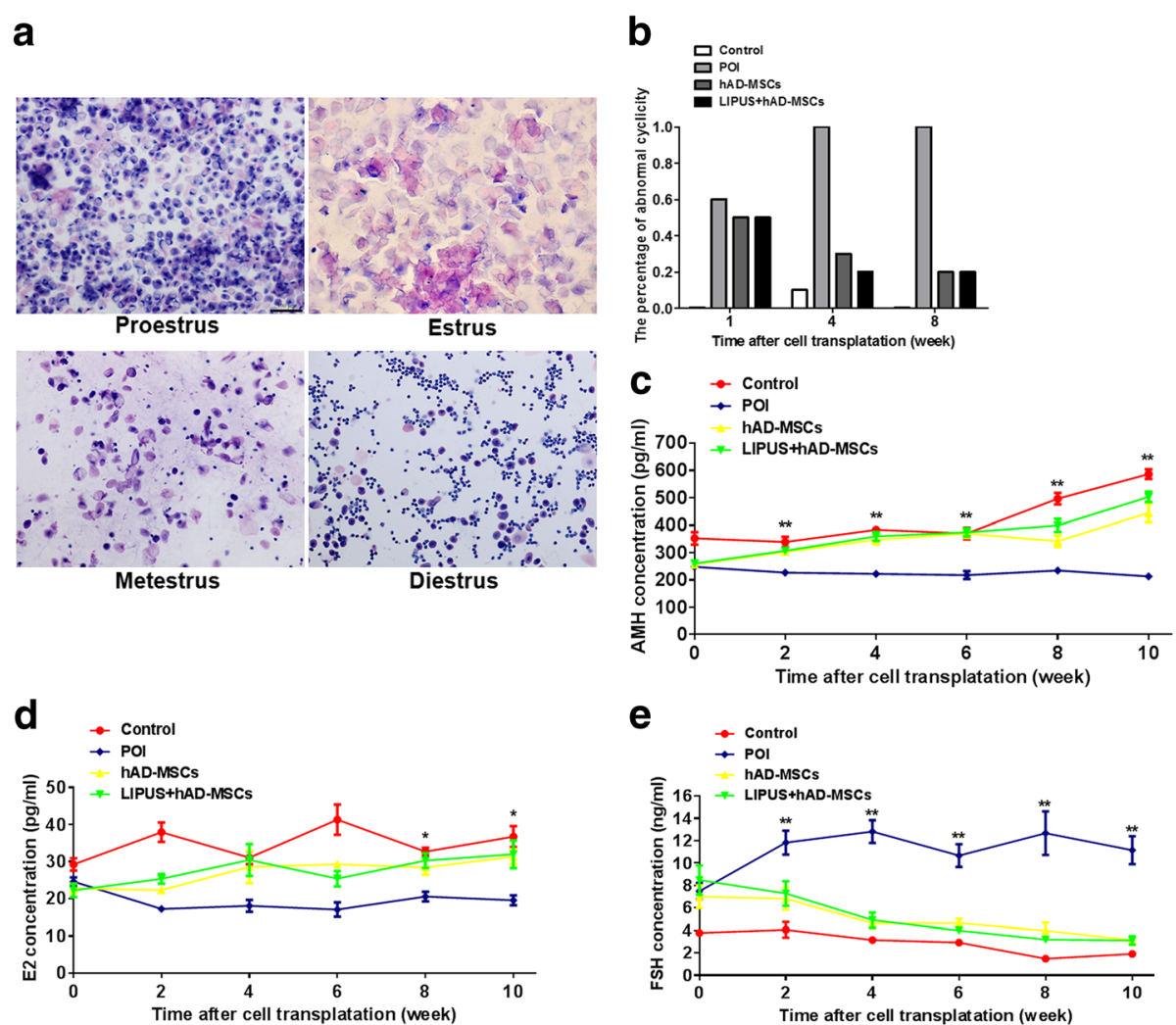

Fig. 5 Transplantation of hAD-MSCs improves ovarian function in POI rats. a Vaginal smears were obtained and the estrous cycles were evaluated by H\&E staining. Representative photographs for estrus, proestrus, metestrus, and diestrus are shown (100X). $\mathbf{b}$ The percentage of rats with abnormal cyclicity was detected at 1, 4, and 8 weeks after cell transplantation. c-e Serum levels of AMH (c), E2 (d), and FSH (e) were detected at 0, 2, 4, 6, 8, and 10 weeks after cell transplantation. Scale bars $=100 \mu \mathrm{m} .{ }^{*} P<0.05,{ }^{* *} P<0.01$. AMH anti-Müllerian hormone, FSH follicle-stimulating hormone, $h A D-M S C S$ human adipose-derived mesenchymal stem cells, LIPUS low-intensity pulsed ultrasound, $P O /$ primary ovarian insufficiency

secondary, and preovulatory follicles in the POI group than in the control group, while there were significantly more atretic follicles in the POI group than in the control group $(P<0.05$; Fig. 6a and $\mathrm{c})$. Moreover, compared to the POI group, the number of follicles at various stages was significantly increased in both the hADMSCs and LIPUS + hAD-MSCs groups at 1 month after cell transplantation $(P<0.05$; Fig. $6 \mathrm{a}$ and $\mathrm{c})$, while the number of atretic follicles was significantly decreased in the hAD-MSCs and LIPUS + hAD-MSCs groups. However, there was no significant difference between the hAD-MSCs and LIPUS + hAD-MSCs groups. The main pathological change in the uterus in the POI group was the atrophy of uterine endometrium and myometrium due to a lack of estrogen (Fig. 6b). These changes were improved by cell transplantation in the hAD-MSCs and LIPUS + hAD-MSCs groups. However, there was no significant difference between these two groups. Graftversus-host disease was not found in any transplanted rats. No obvious organic lesions were seen in the livers, hearts, lungs, spleens, or kidneys after chemotherapy. Taken together, these results demonstrate that both
hAD-MSCs and LIPUS-pretreated hAD-MSC transplantation can reduce the injuries to reproductive organs in the POI rats

\section{Transplantation of hAD-MSCs reduces ovarian GC apoptosis in $\mathrm{POI}$ rats}

To explore the effects of hAD-MSC transplantation on ovarian cell apoptosis induced by chemotherapy, TUNEL staining was used at 1 month after cell transplantation. Our results show that a large number of apoptotic GCs in ovaries were observed in the POI group. The number of apoptotic GCs in both the hAD-MSCs and LIPUS + hAD-MSCs groups was significantly less than in the POI group. Moreover, the number of apoptotic GCs in the LIPUS + hAD-MSCs group was less than in the hADMSCs group (Fig. 7a). The expression levels of apoptosis-related proteins, i.e., Bax and $\mathrm{Bcl}-2$, in the ovaries were detected and quantified using Western blot analysis (Fig. 7b and c). Our results show that, compared to the control group, the levels of Bax were significantly increased $(P<0.01)$ while the levels of Bcl-2 were significantly decreased $(P<0.01)$ in the POI group. On the 
a
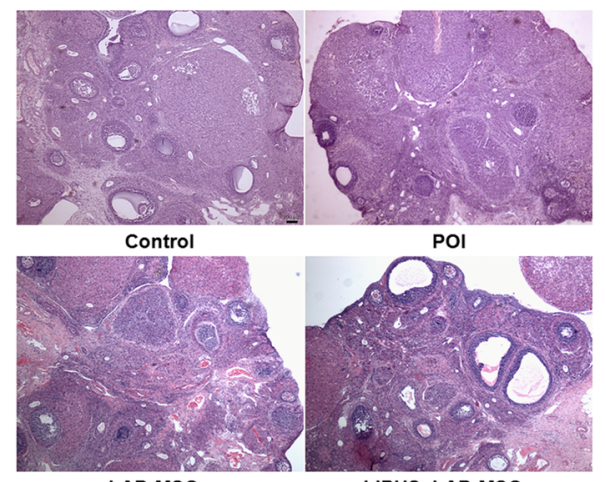

hAD-MSCs

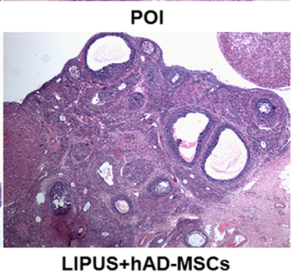

LIPUS+hAD-MSCs b
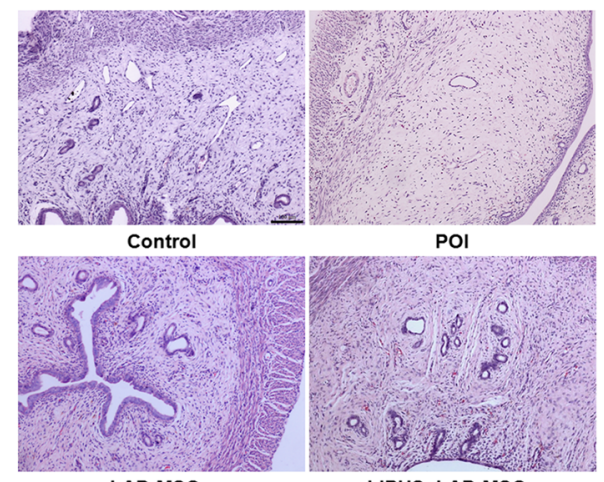

LIPUS+hAD-MSCs

C

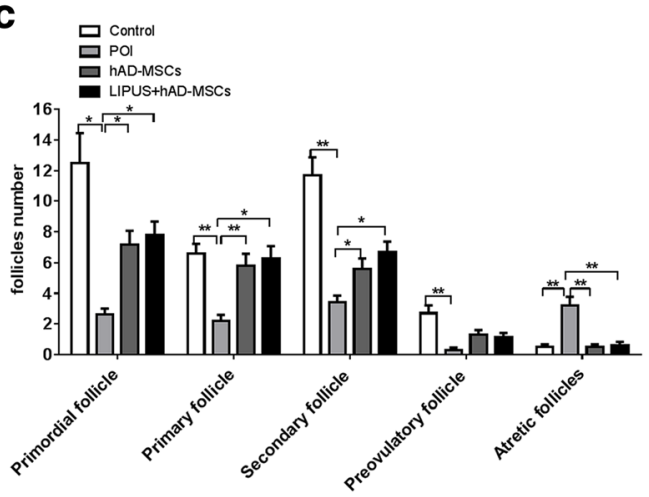

Fig. 6 Transplantation of hAD-MSCs reduces reproductive organ injuries in POI rats. a,b The pathological changes of ovaries (a; 40X) and uteruses (b; 100x) were evaluated by H\&E staining in the four groups at 1 month after hAD-MSC transplantation. c The number of follicles at various stages were counted and compared in the four groups. Scale bars $=100 \mu \mathrm{m} .{ }^{*} P<0.05,{ }^{*} P<0.01$. hAD-MSCs human adipose-derived mesenchymal stem cells, LIPUS low-intensity pulsed ultrasound, $P O I$ primary ovarian insufficiency

other hand, compared to the POI group, the levels of Bax were significantly decreased while the levels of $\mathrm{Bcl}-2$ were significantly increased by cell transplantation in both the hAD-MSCs and LIPUS + hAD-MSCs groups $(P<0.01)$. The $\mathrm{Bcl}-2 / \mathrm{Bax}$ ratio in the POI group was significantly lower than in the other three groups (all $P<0.01)$. Compared to the POI group, the hAD-MSC transplantation significantly increased the Bcl-2/Bax ratio in the hAD-MSCs and LIPUS + hAD-MSCs groups $(P<0.01)$. Moreover, the $\mathrm{Bcl}-2 / \mathrm{Bax}$ ratio in the LIPUS + hAD-MSCs group was significantly higher than in the hAD-MSCs group $(P<0.01)$. These results demonstrate that hAD-MSCs can reduce the ovarian GC apoptosis and increase the $\mathrm{Bcl}-2 / \mathrm{Bax}$ ratio in POI rats, and the effect could be improved by pre-treatment with LIPUS.

\section{Transplantation of hAD-MSCs reduces ovarian inflammation induced by chemotherapy}

To determine the effect of hAD-MSC transplantation on ovarian inflammation induced by chemotherapy in POI rats, the levels of pro-inflammatory cytokines and VEGF in the ovaries were detected (Fig. 8). It was found that, compared to the control group, the levels of several pro- inflammatory cytokines (IL-1 $\beta$, IL-6, and TNF- $\alpha$ ) were significantly increased $(P<0.01)$ while the VEGF level was significantly decreased $(P<0.01)$ in the POI group. Compared to the POI group, the levels of pro-inflammatory cytokines were significantly decreased $(P<0.01)$ while the VEGF levels were significantly increased $(P<0.01)$ after hAD-MSC transplantation in both the hAD-MSCs and LIPUS + hAD-MSCs groups. Moreover, the levels of IL-6 and TNF- $\alpha$ in the LIPUS + hAD-MSCs group were significantly lower than in the hAD-MSCs group $(P<0.05)$, while the VEGF level in the LIPUS + hAD-MSCs group was significantly higher than in the hAD-MSCs group $(P<0.05)$. These results demonstrate that hAD-MSCs can reduce ovarian inflammation induced by chemotherapy and increase the expression of VEGF in ovarian tissue, and that this effect could be improved by pre-treatment with LIPUS.

\section{Discussion}

In our study the results showed that hAD-MSCs isolated from the human amnion had the common characteristics of multipotent MSCs, and the procedure to obtain hAD-MSCs was noninvasive, safe, and out of ethical 

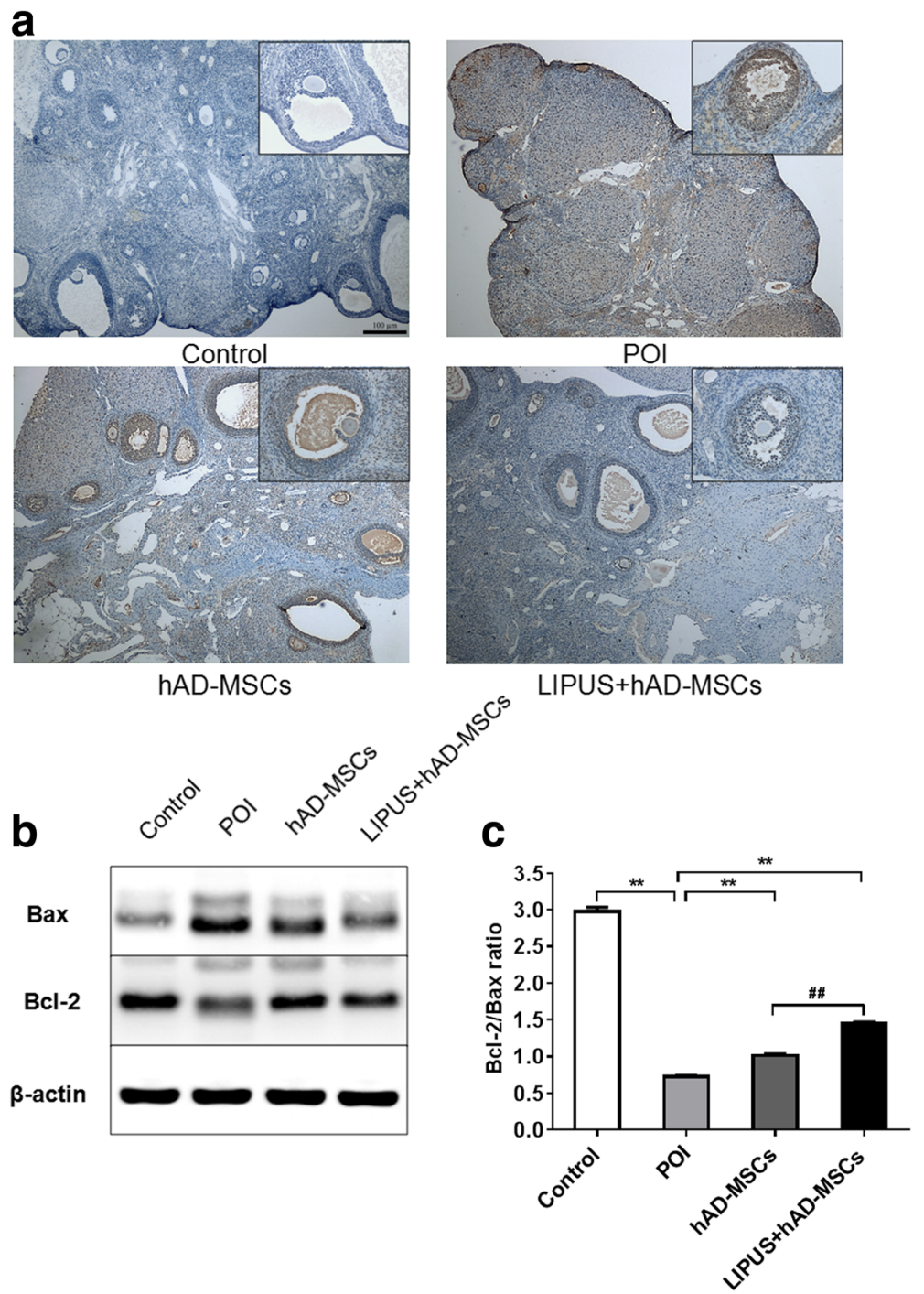

Fig. 7 Transplantation of hAD-MSCs reduces ovarian CG apoptosis in POI rats. a Dark brown cells indicate the ovarian apoptotic cells stained by TUNEL apoptosis assay kit (40X and 200X). b,c Bax and Bcl-2 expression levels were determined by Western blot analysis (b), and the Bcl-2/Bax ratios were evaluated $(\mathbf{c})$. Scale bars $=100 \mu \mathrm{m}$. ${ }^{*} P<0.05,{ }^{*} P<0.01 ;{ }^{\# \#} P<0.01$. hAD-MSCS human adipose-derived mesenchymal stem cells, LIPUS low-intensity pulsed ultrasound, $P O /$ primary ovarian insufficiency

debate, consistent with previous studies [10-12]. In our experiments, a large number of hAD-MSCs can be isolated from human term amnion and expanded rapidly in vitro. The xenotransplantation of hAD-MSCs did not induce xenogeneic immune responses in rats with POI, suggesting the low immunogenicity of hAD-MSCs. These results demonstrate that hAD-MSCs may represent promising seed cells for tissue engineering and clinical applications in the near future.

Ovarian dynamics are characterized by repeated proliferation and differentiation of follicular cells, which is regulated by steroids, growth hormones, gonadotrophins, and a variety of intra-ovarian growth factors, including IGF, FGF2, VEGF, and HGF [39]. LIPUS is a form of mechanical vibration energy transmission. The acoustic pressure wave produced by LIPUS is able to transmit into and through living cells, which may result in a series of biochemical events at the cellular level [17, 18]. In contrast to high-intensity continuous ultrasound, LIPUS is a pulsed emission with low-intensity and low thermal effect, with minimal or no adverse effects on cells [40]. Several studies have found that LIPUS can enhance the production of VEGF in human mandibular osteoblasts, human peripheral blood monocytes, and human osteoblasts [41, 42]. Doan et al. [41] found that LIPUS treatment can upregulate the production of FGF2 in human mandibular osteoblasts. Moreover, Naruse et al. $[43,44]$ found that LIPUS can upregulate IGF-1 in 


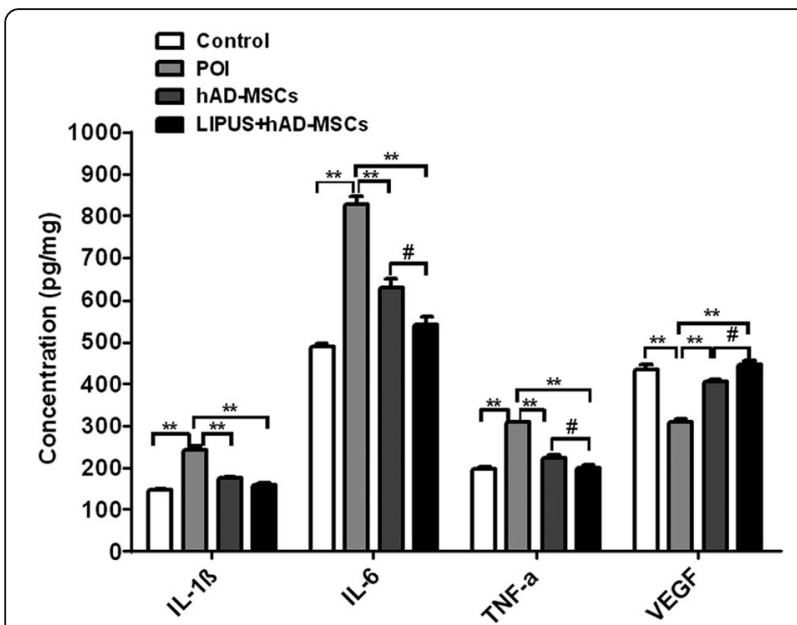

Fig. 8 Transplantation of hAD-MSCs reduces ovary inflammation induced by chemotherapy. Expression levels of pro-inflammatory cytokines (IL-1 $\beta, I L-6$, and TNF- $\alpha$ ) and VEGF in the ovaries were detected by ELISA assay. ${ }^{*} P<0.05$, ${ }^{* *} P<0.01$; ${ }^{*} P<0.05$. hADMSCs human adipose-derived mesenchymal stem cells, IL interleukin, LIPUS low-intensity pulsed ultrasound, $P O /$ primary ovarian insufficiency, TNF tumor necrosis factor, VEGF vascular endothelial growth factor

rat osteoblasts and the murine ST2 bone marrowderived cell line. These growth factors are crucial for angiogenesis and follicle growth in the ovary, which inhibits GC apoptosis [26-29]. In our experiments, our results showed that LIPUS could promote the gene and protein expression of IGF-1, HGF, and VEGF in hADMSCs, which suggested that transplantation of LIPUSpretreated hAD-MSCs might be more advantageous to treat patients with POI.

In this study, the effects of hAD-MSCs and LIPUSpretreated hAD-MSCs on chemotherapy-induced POI were explored. Our results showed that both hAD-MSC and LIPUS-pretreated hAD-MSC transplantation dramatically increased the body and reproductive organ weights of POI rats, and repaired ovarian injury and improved ovarian function. Although hAD-MSCs successfully recovered the ovarian function in rats with POI, they were only found in the interstitium of ovaries and did not differentiate into follicle components after transplantation. The results indicate that hAD-MSCs may restore ovarian function of chemotherapy-induced POI through paracrine effects, rather than through differentiating into GCs or oocytes. Previous studies have found that MSCs can inhibit cell apoptosis and enhance cell survival through paracrine pathways [14, 45], which thus exert positive effects on repairing ovarian tissue damage $[8,36]$. It has been proven that human MSCs can secrete various cytokines, such as IL-6, IL-8, IL-10, IL-11, IL-15, VEGF, granulocyte colony-stimulating factor (G-CSF), HGF, IGF-1, and FGF2 [46, 47]. In our study, we focused on FGF2, IGF-1, HGF, and VEGF, which are likely to play important roles in restoring ovarian function in POI animals via stem cells $[7,46,47]$. It was found that hAD-MSCs can secrete FGF2, IGF-1, HGF, and VEGF, and the expression and secretion of IGF-1, HGF, and VEGF in hAD-MSCs could be promoted by LIPUS treatment. Our results further showed that, although both hAD-MSCs and LIPUS-pretreated hAD-MSCs could reduce inflammation, improve local microenvironment, and inhibit GC apoptosis induced by chemotherapy in ovarian tissue, the efficacy of LIPUS-pretreated hADMSCs were superior to hAD-MSCs. Thus, we speculate that the repaired ovarian tissue damage and improved ovarian function are more likely to be partially mediated by those growth factors produced by hAD-MSCs through the paracrine pathway.

As we know, scholars have verified the existence of ovarian germ stem cells, which can differentiate into reproductive cells and primitive GCs to replenish the follicle pool $[48,49]$. The stem cell microenvironment, also called the stem cell niche, plays an important role in tissue repair after injury. The germ stem cell niche is composed of cytokines, extracellular matrix, and niche cells (mainly including GCs and vascular endothelial cells) surrounding the ovarian germ stem cells [50,51]. Studies have showed that ovarian function failure is mainly due to the aging of the ovarian germ stem cell niches but not due to the aging of the ovarian germ stem cells [51-53]. Scholars have successfully separated ovarian germ stem cells from the ovaries of mice and humans with POI [51, 54], and ovarian germ stem cells from POI ovaries have the ability to differentiate into oocyte in vitro [54]. Thus, the microenvironment in ovarian tissue plays an important role in ovarian function failure and aging [51], and improving the microenvironment for ovaries of POI might make the ovarian germ stem cells remain active and continue to replenish the follicle pool. Winkler et al. found that CTX injured the stem cell niche [55]. Sun et al. found that transplantation of BM-MSCs was effective in inducing osteoblastic niche reconstruction and improving the hematopoietic stem cell niche [56]. Studies showed that CTX broke the inflammatory balance in ovaries through inhibiting the production of the antiinflammatory cytokines and increasing the production of the pro-inflammatory cytokines $[57,58]$ and induced the apoptosis of GCs [25, 57], which is consistent with our results. Cytokines, GCs, and vascular endothelial cells are important components of the germ stem cell niche. Our studies found that hAD-MSC or LIPUS-pretreated hAD-MSC transplantation downregulated the expression of pro-inflammatory cytokines (IL-1 $\beta$, IL- 6 , and TNF- $\alpha$ ), which thus attenuated ovarian inflammation induced by CTX, upregulated VEGF, and reduced the ovarian GC apoptosis in the ovaries of POI rats. VEGF is an effective 
mitogen for vascular endothelial cells and a critical cytokine for angiogenesis [59]. Improving VEGF expression in the ovary is helpful for ovarian angiogenesis, GC survival, and follicle growth [60,61]. Thus, hAD-MSC or LIPUS-pretreated hAD-MSC transplantation might improve the germ stem cell niche. Additionally, it is reported that MSC transplantation reduced the depletion of germ stem cells induced by CTX [4]. From these results, we speculate that hAD-MSC transplantation might reduce the depletion of ovarian germ stem cells and improve the germ stem cell niches through paracrine pathways, which might promote ovarian germ stem cells to differentiate into primordial follicles. On the other hand, Lai et al. also showed that MSC transplantation further reduced depletion of follicles at various stages after chemotherapy, including primordial follicles [4]. In this study, we found that the number of primordial follicles increased in both the hAD-MSCs and LIPUS + hADMSCs groups. Our results showed that the efficacy of LIPUS-pretreated hAD-MSCs was superior to hADMSCs in improving the ovarian microenvironment in rats with POI. The number of primordial follicles in the LIPUS + hAD-MSCs group increased more than in the hAD-MSCs group. However, there was no significant difference between these two groups. Further research is needed to investigate the mechanism underlying the effect of LIPUS + hAD-MSCs and hAD-MSCs on primordial follicles.

The POI rat model was established using CTX, which has been found to be able to cause POI in humans [25]. Moreover, CTX could induce apoptosis of GCs [25] which are required for oocyte survival and follicle development [62, 63]. In this study, our results found that GC apoptosis was significantly increased in the POI group as CTX was administered to the rats. After hAD-MSC or LIPUS-pretreated hAD-MSC transplantation, hADMSCs or LIPUS-pretreated hAD-MSCs dramatically reduced the ovarian GC apoptosis in the developing follicles, and the LIPUS-pretreated hAD-MSCs were found to be more advantageous in reducing GC apoptosis. Regulation of apoptosis is a very complex process, mainly involving two signaling transduction pathways: the extrinsic and intrinsic pathways. The extrinsic pathway starts at the cell surface by activating the cell death receptors, including the tumor necrosis factor receptor (TNFR). TNFR receives the death signal from TNF- $\alpha$, which initiates a pathway that finally leads to apoptosis. In our study, our results showed that both hAD-MSCs and LIPUS-pretreated hAD-MSCs can downregulate the expression of TNF- $\alpha$ in POI ovaries, and the expression of TNF- $\alpha$ in the LIPUS + hAD-MSCs group was significantly lower than in the hAD-MSCs group. The results showed that hAD-MSCs may inhibit ovarian GC apoptosis partially through reducing the expression of TNF- $\alpha$ in ovaries, and this effect may be promoted by pretreatment with LIPUS on hAD-MSCs. On the other hand, the intrinsic pathway starts from the mitochondria, which can be activated by chemotherapy. Activation of the intrinsic pathway is primarily regulated by the $\mathrm{Bcl}-2$ family, among which the pro- and antiapoptotic molecules (Bax and Bcl-2) are the most common markers for apoptosis [64]. Hussein et al. [65] found that one of the primary mechanisms involved in ovarian cell apoptosis is the intrinsic pathway, which is mediated by the $\mathrm{BCl}-2$ family members. $\mathrm{Bcl}-2 / \mathrm{Bax}$ acts as a regulator in the follicular maturation and atresia. The $\mathrm{Bcl}-2 / \mathrm{Bax}$ ratio plays an important role in regulating ovarian cell apoptosis, which determines the fate of ovarian GCs [66]. Thus, we explored the expression of Bax and $\mathrm{Bcl}-2$ proteins in our four groups. Our results showed that ovarian injury induced by chemotherapy resulted in increased Bax expression and decreased Bcl-2 expression, which reduced the $\mathrm{Bcl}-2 / \mathrm{Bax}$ ratio in $\mathrm{POI}$ rats. Both hAD-MSC and LIPUS-pretreated hAD-MSC transplantation significantly reduced the Bax expression and significantly increased the Bcl-2 expression and the $\mathrm{Bcl}-2 / \mathrm{Bax}$ ratio in the ovaries of POI rats. These results indicate that hAD-MSCs can protect ovarian cells against apoptosis by upregulating the $\mathrm{Bcl}-2 / \mathrm{Bax}$ ratio and that they play an anti-apoptotic role in POI.

Previous studies have shown that growth factors can induce the expression of anti-apoptotic $\mathrm{Bcl}-2$ family members $[67,68]$ and reduce the expression of proapoptotic Bax [26]. In our study, LIPUS-pretreated hAD-MSCs showed the benefit of increased Bcl-2/Bax ratio and anti-apoptotic effects compared with the hADMSCs. Thus, we speculate that the mechanisms may be attributed to the increased growth factors in hAD-MSCs promoted by LIPUS, which increases the Bcl-2/Bax ratio; this needs further studies to confirm in the future.

\section{Conclusion}

In conclusion, hAD-MSCs have the common characteristics of multipotent MSCs. Both hAD-MSC and LIPUSpretreated hAD-MSC transplantation could repair ovarian injury and improve ovarian function in rats with chemotherapy-induced POI. LIPUS-pretreated hADMSC transplantation is more advantageous in reducing inflammation and inhibiting GC apoptosis induced by chemotherapy in the ovarian tissue. The efficacy of hAD-MSCs is more likely to be partially mediated by growth factors produced by hAD-MSCs through the paracrine pathway. These findings suggest that LIPUS may provide a way to promote the hAD-MSC secretome, and may be useful in MSC transplantation or a cell-free therapeutic approach (absence of stem cells) for the disease treatment in the clinic. 


\section{Abbreviations}

AMH: Anti-Müllerian hormone; ANOVA: Analysis of variance; BM-MSC: Bone marrow-derived mesenchymal stem cell; CCK-8: Cell counting kit-8; CTX: Cyclophosphamide; DAPI: 2-(4-amidinophenyl)-6-indolecarbamidine dihydrochloride; EDTA: Ethylenediaminetetraacetic acid; ELISA: Enzyme-linked immunosorbent assay; FDA: Food and Drug Administration; FGF: Fibroblast growth factor; FITC: Fluorescein isothiocyanate; FSH: Follicle-stimulating hormone; GC: Granulosa cell; H\&E: Hematoxylin and eosin; hAD-MSC: Human amnion-derived mesenchymal stem cell; HGF: Hepatocyte growth factor; IGF: Insulin-like growth factor; IL: Interleukin; LIPUS: Low-intensity pulsed ultrasound; MSC: Mesenchymal stem cell; OCT: Optimal cutting temperature; PE: Phycoerythrin; POF: Premature ovarian failure; POI: Primary ovarian insufficiency; RT-qPCR: Real-time quantitative polymerase chain reaction; SD: Sprague-Dawley; TNF: Tumor necrosis factor; TNFR: Tumor necrosis factor receptor; VEGF: Vascular endothelial growth factor

\section{Acknowledgments}

We thank the State Key Laboratory of Ultrasound Engineering in Medicine Co-Founded by Chongqing and the Ministry of Science and Technology, Chongqing Key Laboratory of Biomedical Engineering and Laboratory of Stem Cell and Tissue Engineering of Chongqing Medical University for their technical assistance.

\section{Funding}

This work was supported by the National Natural Science Foundation of China (no. 81671415).

\section{Availability of data and materials}

All data and materials are available in the manuscript.

\section{Authors' contributions}

Conceived and designed the experiments: $L L, Z X$; literature search: $L L, L H$, TW, XF; performed the experiments: LL, TW, LH, XF, WZ, ZW, QZ; analyzed the data: $L L, Y W, Z X$; provided ultrasonic equipment and technical support: YW; helped perform the analysis with constructive discussions: $L L, Z X, Y W$, YW, XF; drafted the manuscript: LL. All authors read and approved the final manuscript.

\section{Ethics approval and consent to participate}

The research was in compliance with the Helsinki Declaration and approved by the Ethics Committee of the Second Affiliated Hospital of Chongqing Medical University. Written informed consent was obtained from all donors before collecting amnion. Animal experimental protocols were approved by the Ethics Committee of the Second Affiliated Hospital of Chongqing Medical University (permit number 2016-044).

\section{Consent for publication}

Not applicable.

\section{Competing interests}

The authors declare that they have no competing interests.

\section{Publisher's Note}

Springer Nature remains neutral with regard to jurisdictional claims in published maps and institutional affiliations.

\footnotetext{
Author details

${ }^{1}$ Department of Obstetrics and Gynecology, the Second Affiliated Hospital of Chongqing Medical University, No. 76, Linjiang Road, Chongqing 400010, China. ${ }^{2}$ State Key Laboratory of Ultrasound Engineering in Medicine Co-Founded by Chongqing and the Ministry of Science and Technology, Chongqing Key Laboratory of Biomedical Engineering, College of Biomedical Engineering, Chongqing Medical University, Chongqing 400010, China. ${ }^{3}$ Department of Histology and Embryology, Laboratory of Stem Cell and Tissue Engineering, Chongqing Medical University, Chongqing 400010, China. ${ }^{4}$ Department of Obstetrics and Gynecology, the Third Affiliated Hospital, Zunyi Medical College, Zunyi 563000, Guizhou, China.
}

Received: 9 October 2017 Revised: 26 November 2017

Accepted: 29 November 2017 Published online: 19 December 2017

\section{References}

1. Cho SH, Ahn EH, An HJ, Kim JH, Ko JJ, Kim YR, et al. Association of miR938G > A polymorphisms with primary ovarian insufficiency (POI)-related gene expression. Int J Mol Sci. 2017;18:1255

2. Sullivan SD, Sarrel PM, Nelson LM. Hormone replacement therapy in young women with primary ovarian insufficiency and early menopause. Fertil Steril. 2016;106:1588-99.

3. European Society for Human Reproduction and Embryology (ESHRE) Guideline Group on POI, Webber L, Davies M, Anderson R, Bartlett J, et al. ESHRE Guideline: management of women with premature ovarian insufficiency. Hum Reprod. 2016;31:926-37.

4. Lai D, Wang F, Yao X, Zhang Q, Wu X, Xiang C. Human endometrial mesenchymal stem cells restore ovarian function through improving the renewal of germline stem cells in a mouse model of premature ovarian failure. J Transl Med. 2015;13:155.

5. Park MR, Choi YJ, Kwon DN, Park C, Bui HT, Gurunathan S, et al. Intraovarian transplantation of primordial follicles fails to rescue chemotherapy injured ovaries. Sci Rep. 2013;3:1384

6. Meirow D, Dor J, Kaufman B, Shrim A, Rabinovici J, Schiff E, et al. Cortical fibrosis and blood-vessels damage in human ovaries exposed to chemotherapy. Potential mechanisms of ovarian injury. Hum Reprod. 2007; 22:1626-33

7. Yao X, Guo Y, Wang Q, Xu M, Zhang Q, Li T, et al. The paracrine effect of transplanted human amniotic epithelial cells on ovarian function improvement in a mouse model of chemotherapy-induced primary ovarian insufficiency. Stem Cells Int. 2016;2016:4148923.

8. Wang Z, Wang Y, Yang T, Li J, Yang X. Study of the reparative effects of menstrual-derived stem cells on premature ovarian failure in mice. Stem Cell Res Ther. 2017:8:11.

9. Wang S, Yu L, Sun M, Mu S, Wang C, Wang D, et al. The therapeutic potential of umbilical cord mesenchymal stem cells in mice premature ovarian failure. Biomed Res Int. 2013;2013:690491.

10. Diaz-Prado S, Muinos-Lopez E, Hermida-Gomez T, Rendal-Vazquez ME, Fuentes-Boquete I, de Toro FJ, et al. Multilineage differentiation potential of cells isolated from the human amniotic membrane. J Cell Biochem. 2010; 111:846-57.

11. Soncini M, Vertua E, Gibelli L, Zorzi F, Denegri M, Albertini A, et al. Isolation and characterization of mesenchymal cells from human fetal membranes. J Tissue Eng Regen Med. 2007;1:296-305.

12. Parolini $\mathrm{O}$, Alviano $F$, Bagnara GP, Bilic G, Buhring $H J$, Evangelista $M$, et al. Concise review: isolation and characterization of cells from human term placenta: outcome of the first international workshop on placenta derived stem cells. Stem Cells. 2008;26:300-11.

13. Malek A, Bersinger NA. Human placental stem cells: biomedical potential and clinical relevance. J Stem Cells. 2011;6:75-92.

14. Miyamoto S, Ohnishi S, Onishi R, Tsuchiya I, Hosono H, Katsurada T, et al. Therapeutic effects of human amnion-derived mesenchymal stem cell transplantation and conditioned medium enema in rats with trinitrobenzene sulfonic acid-induced colitis. Am J Transl Res. 2017:9:940-52.

15. Tuca AC, Ertl J, Hingerl K, Pichlsberger M, Fuchs J, Wurzer P, et al. Comparison of Matrigel and Matriderm as a carrier for human amnion-derived mesenchymal stem cells in wound healing. Placenta. 2016;48:99-103.

16. Zhou H, Zhang H, Yan Z, Xu R. Transplantation of human amniotic mesenchymal stem cells promotes neurological recovery in an intracerebral hemorrhage rat model. Biochem Biophys Res Commun. 2016;475:202-8.

17. Padilla F, Puts R, Vico L, Raum K. Stimulation of bone repair with ultrasound: a review of the possible mechanic effects. Ultrasonics. 2014:54:1125-45.

18. Hu B, Zhang Y, Zhou J, Li J, Deng F, Wang Z, et al. Low-intensity pulsed ultrasound stimulation facilitates osteogenic differentiation of human periodontal ligament cells. PLoS One. 2014;9:e95168.

19. LV Y, Zhao P, Chen G, Sha Y, Yang L. Effects of low-intensity pulsed ultrasound on cell viability, proliferation and neural differentiation of induced pluripotent stem cells-derived neural crest stem cells. Biotechnol Lett. 2013:35:2201-12.

20. Harrison A, Lin S, Pounder N, Mikuni-Takagaki Y. Mode and mechanism of low intensity pulsed ultrasound (LIPUS) in fracture repair. Ultrasonics. 2016; 70:45-52. 
21. Sena K, Angle SR, Kanaji A, Aher C, Karwo DG, Sumner DR, et al. Lowintensity pulsed ultrasound (LIPUS) and cell-to-cell communication in bone marrow stromal cells. Ultrasonics. 2011;51:639-44.

22. Coords M, Breitbart E, Paglia D, Kappy N, Gandhi A, Cottrell J, et al. The effects of low-intensity pulsed ultrasound upon diabetic fracture healing. J Orthop Res. 2011;29:181-8.

23. Sen B, Xie Z, Case N, Thompson WR, Uzer G, Styner M, et al. mTORC2 regulates mechanically induced cytoskeletal reorganization and lineage selection in marrow-derived mesenchymal stem cells. J Bone Miner Res. 2014:29:78-89.

24. Wang J, Wang CD, Zhang N, Tong WX, Zhang YF, Shan SZ, et al. Mechanical stimulation orchestrates the osteogenic differentiation of human bone marrow stromal cells by regulating HDAC1. Cell Death Dis. 2016;7:e2221.

25. Bedoschi G, Navarro PA, Oktay K. Chemotherapy-induced damage to ovary: mechanisms and clinical impact. Future Oncol. 2016;12:2333-44.

26. Babitha V, Yadav VP, Chouhan VS, Hyder I, Dangi SS, Gupta M, et al. Luteinizing hormone, insulin like growth factor-1, and epidermal growth factor stimulate vascular endothelial growth factor production in cultured bubaline granulosa cells. Gen Comp Endocrinol. 2014;198:1-12.

27. Zachow R, Uzumcu M. The hepatocyte growth factor system as a regulator of female and male gonadal function. J Endocrinol. 2007;195:359-71.

28. Mao J, Smith MF, Rucker EB, Wu GM, McCauley TC, Cantley TC, et al. Effect of epidermal growth factor and insulin-like growth factor I on porcine preantral follicular growth, antrum formation, and stimulation of granulosal cell proliferation and suppression of apoptosis in vitro. J Anim Sci. 2004;82: 1967-75.

29. Uzumcu M, Pan Z, Chu Y, Kuhn PE, Zachow R. Immunolocalization of the hepatocyte growth factor (HGF) system in the rat ovary and the antiapoptotic effect of HGF in rat ovarian granulosa cells in vitro. Reproduction. 2006;132:291-9.

30. Fang ZL, Song XJ, Zhang T, CHEN DX. Isolation, culture and identification of the mesenchymal stem cells derived from human amnion. Acta Academiae Med Zunyi. 2009;32:234-6.

31. Ji G, Liu M, Zhao XF, Liu XY, Guo QL, Guan ZF, et al. NF-kappaB signaling is involved in the effects of intranasally engrafted human neural stem cells on neurofunctional improvements in neonatal rat hypoxic-ischemic encephalopathy. CNS Neurosci Ther. 2015;21:926-35.

32. Pavyde $E$, Maciulaitis $R$, Mauricas M, Sudzius G, Ivanauskaite Didziokiene E, Laurinavicius A, et al. Skeletal muscle-derived stem/progenitor cells: a potential strategy for the treatment of acute kidney injury. Stem Cells Int. 2016;2016:9618480.

33. Zhu HY, Fu XF, Zhuo GS. In vivo tracking of PKH26-labeled human umbilical cord mesenchymal stem cells after transplantation into rats of chemotherapy-induced premature ovarian failure. Progress Obstet Gynecol. 2011;20:92-6.

34. Modo M, Beech JS, Meade TJ, Williams SC, Price J. A chronic 1 year assessment of MRI contrast agent-labelled neural stem cell transplants in stroke. Neuroimage. 2009;47 Suppl 2:T133-42.

35. Talab SS, Kajbafzadeh AM, Elmi A, Tourchi A, Sabetkish S, Sabetkish N, et al. Bladder reconstruction using scaffold-less autologous smooth muscle cell sheet engineering: early histological outcomes for autoaugmentation cystoplasty. BJU Int. 2014;114:937-45.

36. Liu J, Zhang H, Zhang Y, Li N, Wen Y, Cao F, et al. Homing and restorative effects of bone marrow-derived mesenchymal stem cells on cisplatin injured ovaries in rats. Mol Cells. 2014;37:865-72.

37. Myers M, Britt KL, Wreford NG, Ebling FJ, Kerr JB. Methods for quantifying follicular numbers within the mouse ovary. Reproduction. 2004;127:569-80.

38. Song D, Zhong Y, Qian C, Zou Q, Ou J, Shi Y, et al. Human umbilical cord mesenchymal stem cells therapy in cyclophosphamide-induced premature ovarian failure rat model. Biomed Res Int. 2016;2016:2517514.

39. Mishra SR, Bharati J, Rajesh G, Chauhan VS, Taru Sharma G, Bag S, et al. Fibroblast growth factor 2 (FGF2) and vascular endothelial growth factor A (VEGFA) synergistically promote steroidogenesis and survival of cultured buffalo granulosa cells. Anim Reprod Sci. 2017;179:88-97.

40. Bashardoust Tajali S, Houghton P, MacDermid JC, Grewal R. Effects of lowintensity pulsed ultrasound therapy on fracture healing: a systematic review and meta-analysis. Am J Phys Med Rehabil. 2012;91:349-67.

41. Doan N, Reher P, Meghji S, Harris M. In vitro effects of therapeutic ultrasound on cell proliferation, protein synthesis, and cytokine production by human fibroblasts, osteoblasts, and monocytes. J Oral Maxillofac Surg. 1999;57:409-19. discussion 420.
42. Wang FS, Kuo YR, Wang CJ, Yang KD, Chang PR, Huang YT, et al. Nitric oxide mediates ultrasound-induced hypoxia-inducible factor-1alpha activation and vascular endothelial growth factor-A expression in human osteoblasts. Bone. 2004;35:114-23.

43. Naruse K, Miyauchi A, Itoman M, Mikuni-Takagaki Y. Distinct anabolic response of osteoblast to low-intensity pulsed ultrasound. J Bone Miner Res. 2003;18:360-9.

44. Naruse K, Mikuni-Takagaki Y, Azuma Y, Ito M, Oota T, Kameyama K, et al. Anabolic response of mouse bone-marrow-derived stromal cell clone ST2 cells to low-intensity pulsed ultrasound. Biochem Biophys Res Commun. 2000;268:216-20.

45. Souidi N, Stolk M, Seifert M. Ischemia-reperfusion injury: beneficial effects of mesenchymal stromal cells. Curr Opin Organ Transplant. 2013;18:34-43.

46. Wang Z, Wang Y, Yang T, Li J, Yang X. Erratum to: Study of the reparative effects of menstrual-derived stem cells on premature ovarian failure in mice. Stem Cell Res Ther. 2017:8:49.

47. Takehara Y, Yabuuchi A, Ezoe K, Kuroda T, Yamadera R, Sano C, et al. The restorative effects of adipose-derived mesenchymal stem cells on damaged ovarian function. Lab Invest. 2013;93:181-93.

48. Bukovsky A. Cell commitment by asymmetric division and immune system involvement. Prog Mol Subcell Biol. 2007;45:179-204.

49. White YA, Woods DC, Takai Y, Ishihara O, Seki H, Tilly JL. Oocyte formation by mitotically active germ cells purified from ovaries of reproductive-age women. Nat Med. 2012;18:413-21.

50. Lin $\mathrm{H}$. The stem-cell niche theory: lessons from flies. Nat Rev Genet. 2002;3:931-40.

51. Ye H, Zheng T, Li W, Li X, Fu X, Huang Y, et al. Ovarian stem cell nests in reproduction and ovarian aging. Cell Physiol Biochem. 2017;43:1917-25.

52. Massasa E, Costa XS, Taylor HS. Failure of the stem cell niche rather than loss of oocyte stem cells in the aging ovary. Aging (Albany NY). 2010;2:1-2.

53. Niikura $Y$, Niikura T, Tilly JL. Aged mouse ovaries possess rare premeiotic germ cells that can generate oocytes following transplantation into a young host environment. Aging (Albany NY). 2009;1:971-8.

54. Bukovsky A, Caudle MR. Immunoregulation of follicular renewal, selection, POF, and menopause in vivo, vs. neo-oogenesis in vitro, POF and ovarian infertility treatment, and a clinical trial. Reprod Biol Endocrinol. 2012;10:97.

55. Winkler IG, Pettit AR, Raggatt $L$, Jacobsen RN, Forristal CE, Barbier V, et al. Hematopoietic stem cell mobilizing agents G-CSF, cyclophosphamide or AMD3100 have distinct mechanisms of action on bone marrow HSC niches and bone formation. Leukemia. 2012;26:1594-601.

56. Sun L, Akiyama K, Zhang H, Yamaza T, Hou Y, Zhao S, et al. Mesenchymal stem cell transplantation reverses multiorgan dysfunction in systemic lupus erythematosus mice and humans. Stem Cells. 2009;27:1421-32.

57. Luo Q, Yin N, Zhang L, Yuan W, Zhao W, Luan X, et al. Role of SDF-1/CXCR4 and cytokines in the development of ovary injury in chemotherapy drug induced premature ovarian failure mice. Life Sci. 2017;179:103-9.

58. Lai D, Wang F, Dong Z, Zhang Q. Skin-derived mesenchymal stem cells help restore function to ovaries in a premature ovarian failure mouse model. PLoS One. 2014;9:e98749.

59. Leung DW, Cachianes G, Kuang WJ, Goeddel DV, Ferrara N. Vascular endothelial growth factor is a secreted angiogenic mitogen. Science. 1989; 246:1306-9.

60. Kosaka N, Sudo N, Miyamoto A, Shimizu T. Vascular endothelial growth factor (VEGF) suppresses ovarian granulosa cell apoptosis in vitro. Biochem Biophys Res Commun. 2007:363:733-7.

61. Danforth DR, Arbogast LK, Ghosh S, Dickerman A, Rofagha R, Friedman Cl. Vascular endothelial growth factor stimulates preantral follicle growth in the rat ovary. Biol Reprod. 2003;68:1736-41.

62. Chong JJ, Chandrakanthan V, Xaymardan M, Asli NS, Li J, Ahmed I, Heffernan C, et al. Adult cardiac-resident MSC-like stem cells with a proepicardial origin. Cell Stem Cell. 2011;9:527-40.

63. Iwasaki M, Adachi Y, Minamino K, Suzuki Y, Zhang Y, Okigaki M, et al. Mobilization of bone marrow cells by G-CSF rescues mice from cisplatininduced renal failure, and M-CSF enhances the effects of G-CSF. J Am Soc Nephrol. 2005;16:658-66.

64. Cory S, Adams JM. The Bcl2 family: regulators of the cellular life-or-death switch. Nat Rev Cancer. 2002;2:647-56.

65. Hussein MR, Haemel AK, Wood GS. Apoptosis and melanoma: molecular mechanisms. J Pathol. 2003;199:275-88.

66. He X, Wang SY, Yin CH, Wang T, Jia CW, Ma YM. Hydrogen-rich water exerting a protective effect on ovarian reserve function in a mouse model 
of immune premature ovarian failure induced by zona pellucida 3. Chin Med J (Engl). 2016;129:2331-7.

67. Bonni A, Brunet A, West AE, Datta SR, Takasu MA, Greenberg ME. Cell survival promoted by the Ras-MAPK signaling pathway by transcriptiondependent and -independent mechanisms. Science. 1999;286:1358-62.

68. Datta SR, Brunet A, Greenberg ME. Cellular survival: a play in three Akts. Genes Dev. 1999;13:2905-27.

Submit your next manuscript to BioMed Central and we will help you at every step:

- We accept pre-submission inquiries

- Our selector tool helps you to find the most relevant journal

- We provide round the clock customer support

- Convenient online submission

- Thorough peer review

- Inclusion in PubMed and all major indexing services

- Maximum visibility for your research

Submit your manuscript at www.biomedcentral.com/submit 